\title{
Edge Channel Transport in InAs/GaSb Topological Insulating Phase
}

\author{
Kyoichi Suzuki.* Yuichi Harada, Koji Onomitsu, and Koji Muraki \\ NTT Basic Research Laboratories, NTT Corporation, and \\ 3-1 Morinosato-Wakamiya, Atsugi, Kanagawa 243-0198, Japan
}

(Dated: August 12, 2018)

\begin{abstract}
Transport in InAs/GaSb heterostructures with different InAs layer thicknesses is studied using a six-terminal Hall bar geometry with a $2-\mu \mathrm{m}$ edge channel length. For a sample with a 12-nm-thick InAs layer, non-local resistance measurements with various current/voltage contact configurations reveal that the transport is dominated by edge channels with negligible bulk contribution. Systematic non-local measurements allow us to extract the resistance of individual edge channels, revealing sharp resistance fluctuations indicative of inelastic scattering. Our results show that the InAs/GaSb system can be tailored to have conducting edge channels while keeping a gap in the bulk region and provide a way of studying $2 \mathrm{D}$ topological insulators even when quantized transport is absent.

PACS numbers: 72.25.Dc, 73.63.Hs, 73.61.Ey,
\end{abstract}

\section{INTRODUCTION}

Topological insulators (TIs) have attracted strong interest as a new quantum state of matter categorized neither as a metal nor an insulator ${ }^{1-11}$ Due to their topological nature, gapless states are formed at the sample periphery, i.e., at surfaces and edges in threedimensional ${ }^{4-7}$ and two-dimensional (2D) TIs, ${ }^{8-11}$ respectively. A unique feature of $2 \mathrm{D}$ TIs is that their edge channels are helical; that is, each edge comprises two channels carrying the two spin components in opposite directions. In the absence of a magnetic field or inelastic scattering, transport is protected from backscattering by time-reversal symmetry. If the sample dimension is smaller than the inelastic scattering length, the edge transport becomes dissipationless, which would lead to quantized spin Hall conductance. The observation of a quantum spin Hall effect (QSHE) in $\mathrm{HgTe} / \mathrm{HgCdTe}$ quantum wells 9,10 and subsequent non-local resistance measurements ${ }^{11}$ have confirmed the formation of a $2 \mathrm{D}$ TI phase. However, the experimental demonstration of a 2D TI can be affected by sample disorder, which limits the inelastic mean free path ${ }^{9}-13$ and hence hampers the observation of a QSHE or quantization of non-local transport. Since the existence of edge states in a TI stems from its bulk properties, $\stackrel{4,14}{,}$ a more basic question is whether or not the material has the band structure characteristic of a TI.

Until now, all experimentally confirmed TIs, $,-\underline{4}, 9-11$ such as $\mathrm{Bi}_{1-x} \mathrm{Sb}_{x}, \mathrm{Bi}_{2} \mathrm{Te}_{3}, \mathrm{TlBiSe}_{2}$, and $\mathrm{HgTe}$, naturally have the band structure characteristics of TIs with band inversion caused by spin-orbit interaction. In contrast, the InAs/GaSb hybrid system has been predicted to offer a new type of TI consisting solely of semiconductors with non-inverted band structures and additional band tunability via an electric field 15 For appropriately designed InAs and GaSb layer thicknesses, the bottom of the InAs conduction band becomes lower in energy than the top of the GaSb valence band. Due to band hybridization across the heterointerface and spin-orbit interaction, a small energy gap opens at the crossing points of the orig- inal bands, ${ }^{16-18}$ and helical edge channels are expected to be formed. Recently, Knez and co-workers have investigated InAs/GaSb four-terminal devices. 19 From systematic conductance measurements on samples with various dimensions and length/width aspect ratios, they were able to identify the contribution of edge channel transport with conductance close to the expected quantized value. However, the data indicated significant contributions of bulk transport, making it unfeasible to obtain more direct information about the edge transport.

In this paper, we report transport measurements in the InAs/GaSb system obtained using six-terminal small Hall devices with an edge channel length of $2 \mu \mathrm{m}$. We examine three samples with different InAs layer thicknesses and correspondingly different degrees of band overlap. In addition to the standard longitudinal resistance, we examine non-local resistances for various current/voltage contact configurations. For the optimal InAs layer thickness, the non-local measurements reveal that, over a range of gate voltages, edge channel transport is dominant with negligible bulk contribution. Due to the absence of bulk transport, the non-local measurements allow us to extract the resistances of individual edge channels, revealing reproducible fluctuations indicative of inelastic scattering processes. Our results demonstrate that, by appropriately designing the layer structure, the InAs/GaSb system can be tailored to have conducting edge channels while maintaining a gap in the bulk region, which is characteristic of a $2 \mathrm{D}$ TI band structure. Our method is applicable not only to the InAs/GaSb system but also to other $2 \mathrm{D}$ TI systems including the $\mathrm{HgTe} / \mathrm{HgCdTe}$ system, allowing us to study the properties of edge channel transport ${ }^{20}-24$ even when the inelastic mean free path is shorter than the edge channel length and quantized transport is absent.

\section{SAMPLES}

The layer structure of the samples is shown schematically in Fig. 1(a). InAs/GaSb heterostructures are 
(a)

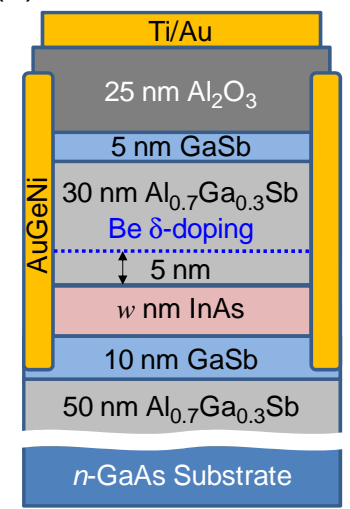

(b)
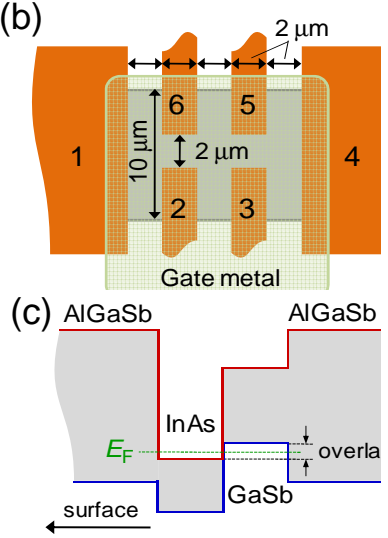

FIG. 1: (Color online) Schematic illustrations of the san (a) Cross-sectional view of layer structure, (b) top view o: bar, and (c) band profile along the growth direction. ? samples with different InAs layer thicknesses $(w=10,12$ $14 \mathrm{~nm}$ ) were examined.

grown by molecular beam epiatxy on an $n^{+}$-GaAs strate, which serves as a back gate, with GaAs/AlAs $\mathrm{GaSb} / \mathrm{AlSb}$ superlattice insulating buffer layers. W amined three heterostructures with different InAs thicknesses $w(=10,12,14 \mathrm{~nm})$. To bring the Fermi close to the energy gap region, the upper $\mathrm{Al}_{0.7} \mathrm{Ga}_{1}$ barrier layer is delta-doped with $[\mathrm{Be}]=5 \times 10^{11} \mathrm{c}$ at a setback of $5 \mathrm{~nm} \cdot{ }^{25}$ Small Hall bar patterns as depicted in Fig. 1(b) are formed by using deep ultraviolet lithography and wet etching. The Hall bars have six ohmic contacts formed by evaporating AuGeNi without annealing. A Ti/Au top gate is evaporated on a $25-\mathrm{nm}$ thick $\mathrm{Al}_{2} \mathrm{O}_{3}$ gate insulating layer deposited by atomic layer deposition ${ }^{26}$ The gate metal entirely covers the active region of the Hall bar, including the portion of each ohmic contact that overlaps the mesa. Ungated regions, if present, would remain $p$-type, which would then form $p$ - $n$ junctions at the boundaries with gated regions when the latter are $n$-type. As a result, when the sample has a relatively large band gap, a gated region would be electrically isolated from the ohmic contacts. To avoid such situations, the gate metal was designed to entirely cover the active region of the Hall bar, including the boundaries with the ohmic contacts. The Hall bars are $10 \mu \mathrm{m}$ wide and the distance between adjacent ohmic contacts is 2 $\mu \mathrm{m}$. Transport measurements are performed at $T=0.25$ $4.3 \mathrm{~K}$ using a lock-in technique with an excitation current of $1 \mathrm{nA}$ and frequency of $13 \mathrm{~Hz}$. All measurements are performed in a zero magnetic field and with a zero backgate voltage.

\section{EFFECTS OF InAs LAYER THICKNESS}

We first show the impact of InAs layer thickness on the transport properties. Figure 2(a) shows longitudinal resistance $R_{x x}\left(=R_{14,23}=V_{23} / I_{14}\right)$ as a function of front
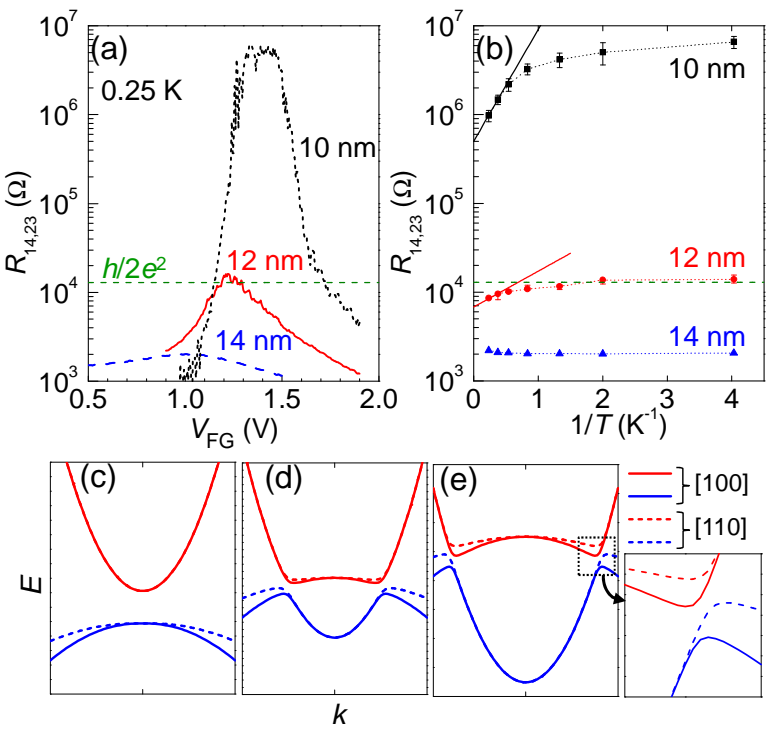

FIG. 2: (Color online) (a) Longitudinal resistance $R_{14,23}$ $\left(=V_{23} / I_{14}\right)$ at $0.25 \mathrm{~K}$ as a function of $V_{\mathrm{FG}}$ for samples with different InAs thicknesses $w$. (b) Temperature dependence of $R_{x x}$ peaks plotted versus inverse temperature $(1 / T)$. Solid lines indicate fits to the high-temperature section of the data, which provide a crude estimate of the gap, $\Delta=0.5$ and 0.2 $\mathrm{meV}$, for $w=10$ and $12 \mathrm{~nm}$, respectively. (c)-(e) Schematic illustrations of energy band diagrams. The band overlap increases from (c) to (e). In (e), the region near the anti-crossing is shown in an enlarged view (right).

gate voltage $\left(V_{\mathrm{FG}}\right)$ for the three samples with different $w$ values measured at $0.25 \mathrm{~K}$. The suffixes $R_{i j, k l}\left(=V_{k l} / I_{i j}\right)$ indicate the contacts used for driving the current $(i, j)$ and measuring the resultant voltage $(k, l)$. A maximum is observed in the $R_{x x}$ trace for all samples, but the peak values differ significantly. The peak resistance varies with $w$ by more than three orders of magnitude, ranging from $2.0 \mathrm{k} \Omega$ for $w=14 \mathrm{~nm}$ up to $8 \mathrm{M} \Omega$ for $w=10 \mathrm{~nm}$. The $w=12 \mathrm{~nm}$ sample shows a peak resistance of $\sim 13 \mathrm{k} \Omega$, which is more than two orders of magnitude smaller than that of the $w=10 \mathrm{~nm}$ sample. Hall measurements at $B$ $=1 \mathrm{~T}$ (data not shown) confirm that, in all the samples, the majority carrier type changes from $p$-type (holes) to $n$-type (electrons) as $V_{\mathrm{FG}}$ is increased across the $R_{x x}$ maximum. In those $V_{\mathrm{FG}}$ ranges away from the $R_{x x} \max -$ imum, the $R_{x x}$ value does not depend significantly on $w$.

The difference in the behavior of these three samples is corroborated by examining the temperature dependence of $R_{x x}$ at its maximum [Fig. 2(b)]. The strongly insulating behavior of the $w=10 \mathrm{~nm}$ sample, with $R_{x x}$ increasing to several $\mathrm{M} \Omega$ with decreasing temperature, clearly shows the existence of a band gap. Fitting the high-temperature section of the data with $R_{x x} \propto \exp \left(\Delta / 2 k_{\mathrm{B}} T\right)\left(k_{\mathrm{B}}\right.$ : Boltzmann constant) provides a crude estimate of the energy gap, $\Delta=0.5 \mathrm{meV}$. On the other hand, the $w=14 \mathrm{~nm}$ sample shows almost no temperature dependence, indicating a gapless band 
structure. In contrast, the $w=12 \mathrm{~nm}$ sample exhibits dual behavior; while the thermally activated behavior in the high temperature regime suggests the existence of a band gap, at low temperatures $R_{x x}$ is saturated and the system remains highly conductive. We note that the peak resistance $\sim 13 \mathrm{k} \Omega$ of the $w=12 \mathrm{~nm}$ sample is close to the quantized value $h / 2 e^{2}$ expected for the QSHE in the present contact geometry (where $h$ is Planck's constant; $e$ is the elementary charge). Actually, the peak resistance for $w=12 \mathrm{~nm}$ varies from 12 to $50 \mathrm{k} \Omega$ among samples fabricated from the same wafer. Even then, by comparison with $w=10 \mathrm{~nm}$ samples, the value is much lower and closer to $h / 2 e^{2}$.

These results can be qualitatively understood from the band diagrams shown schematically in Fig. 1(c) and Figs. 2(c)-2(e). When the InAs layer is thin, due to the strong confinement of the electron wavefunction, the electron subband in InAs lies above the hole subband in GaSb, resulting in a normal semiconductor-like band structure with a band gap [Fig. 2(c)]. As the InAs layer is made thicker, the electron subband becomes lower in energy and, at some point band inversion occurs with the hole subband in GaSb, where band hybridization across the InAs/GaSb interface opens a small gap at the band crossing points [Fig. 2(d)]. Spin-orbit interaction induces linearly dispersing bands inside this gap and they connect the conduction and valence bands, which correspond to the helical edge channels of a $2 \mathrm{D}$ TI ${ }^{15}$ When the Fermi level is in this gap, transport is governed by the helical edge channels at low temperatures. As the InAs layer thickness is increased further, the band overlap increases; however, the influence of the GaSb valence band anisotropy becomes more important because the anti-crossings occur at larger wave numbers $\mathbf{k} \cdot \frac{19}{19}$ While the gap remains open for each $\mathbf{k}$ direction, now the energy position of the gap strongly depends on the $\mathbf{k}$ direction [Fig. 2(e)]. When the anisotropy of the gap position exceeds the size of the gap, the upper and lower bands along different $\mathbf{k}$ directions overlap in energy, leading to a semi-metallic band structure without a band gap.

\section{NON-LOCAL MEASUREMENTS}

To clarify the transport mechanism in the $w=12 \mathrm{~nm}$ sample, we investigate non-local resistances. In a topologically insulating phase, the bulk is insulating and the current would flow only through the helical edge channels that form along the sample edges. The current and voltage distributions in such a topological phase can be understood using the effective circuit model as shown in Fig. 3(a), in which the system can be described as a network of channel resistances $r_{j, j+1}(j=1,2, \ldots ; \bmod$ $6)$. The injected current is partitioned into two paths along the sample edges connecting the source and drain contacts in the clockwise $\left(i_{2}\right)$ and counterclockwise $\left(i_{1}\right)$ directions. In an ideal QSHE system without inelastic scattering, each channel has a quantized conductance of
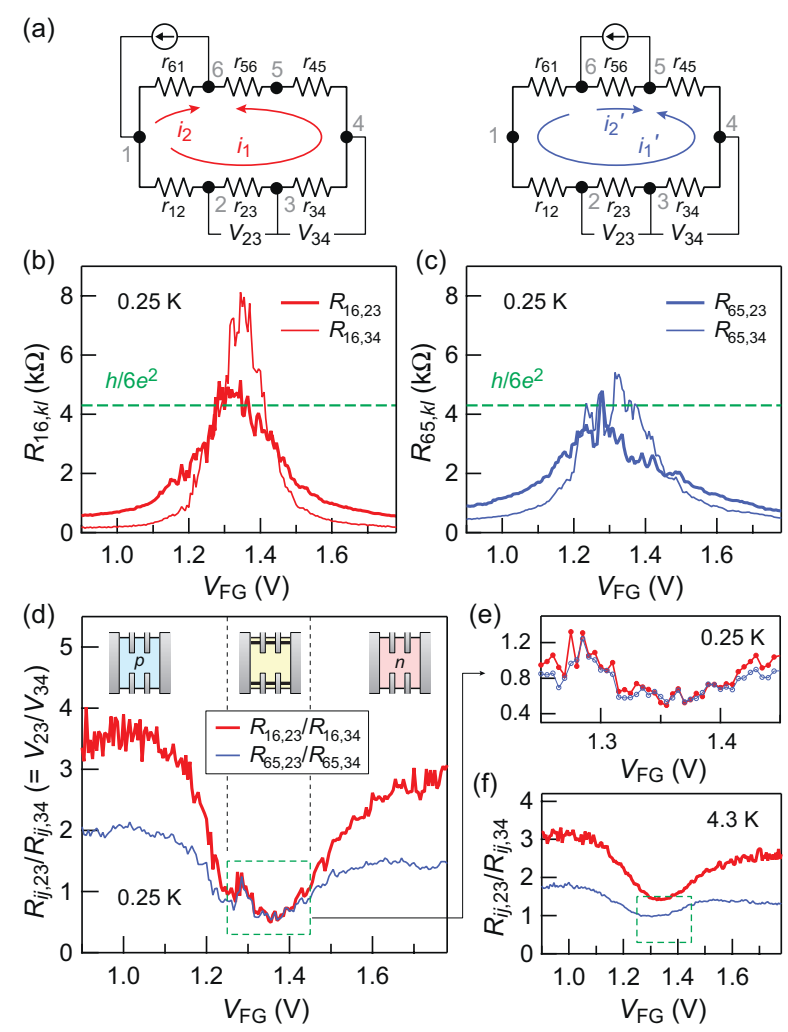

FIG. 3: (Color online) (a) Effective circuit model describing edge transport in the topological insulating phase. Left and right panels represent current/voltage contact configurations for the non-local measurements in (b) and (c), respectively. See main text for details. (b) and (c) non-local resistances of the $w=12 \mathrm{~nm}$ sample at $0.25 \mathrm{~K}$ as a function of $V_{\mathrm{FG}}$ : (b) $R_{16,23}$ and $R_{16,34}$ and (c) $R_{65,23}$ and $R_{65,34}$. (d) Ratio between non-local resistances measured with adjacent voltage-probe pairs (2-3 and 3-4) at $0.25 \mathrm{~K}$. The two traces show results for different current paths (1-6 and 6-5). (e) Magnified view of the data in the dashed rectangle in (d). (f) Similar measurements to $(\mathrm{d})$ at $4.3 \mathrm{~K}$.

$e^{2} / h$ for counterpropagating opposite-spin components, so that the current, when driven through adjacent contacts, is partitioned at a ratio of $1: 5$ in a six-terminal device. Thus, a quantized resistance of $h / 6 e^{2}$ should be observed for the all configurations in an ideal system without inelastic scattering.

Figure 3(b) shows the non-local resistances $R_{16,23}$ and $R_{16,34}$ measured at $0.25 \mathrm{~K}$ as a function of $V_{\mathrm{FG}}$. These two measurements use the same current injection/ejection path (1-6), and differ only in the contact pair (2-3 or 3-4) used for measuring the voltage. Figure 3 (c) shows similar measurements $\left(R_{65,23}\right.$ and $\left.R_{65,34}\right)$ for annother current injection/ejection path (6-5). In the range $1.25<V_{\mathrm{FG}}<1.45 \mathrm{~V}$, where the Fermi level is expected to be in the bulk gap, all the measured nonlocal resistances show a tendency to approach $h / 6 e^{2}$ as expected for the QSHE (indicated by dashed line). However, there are significant deviations from $h / 6 e^{2}$. Intrigu- 


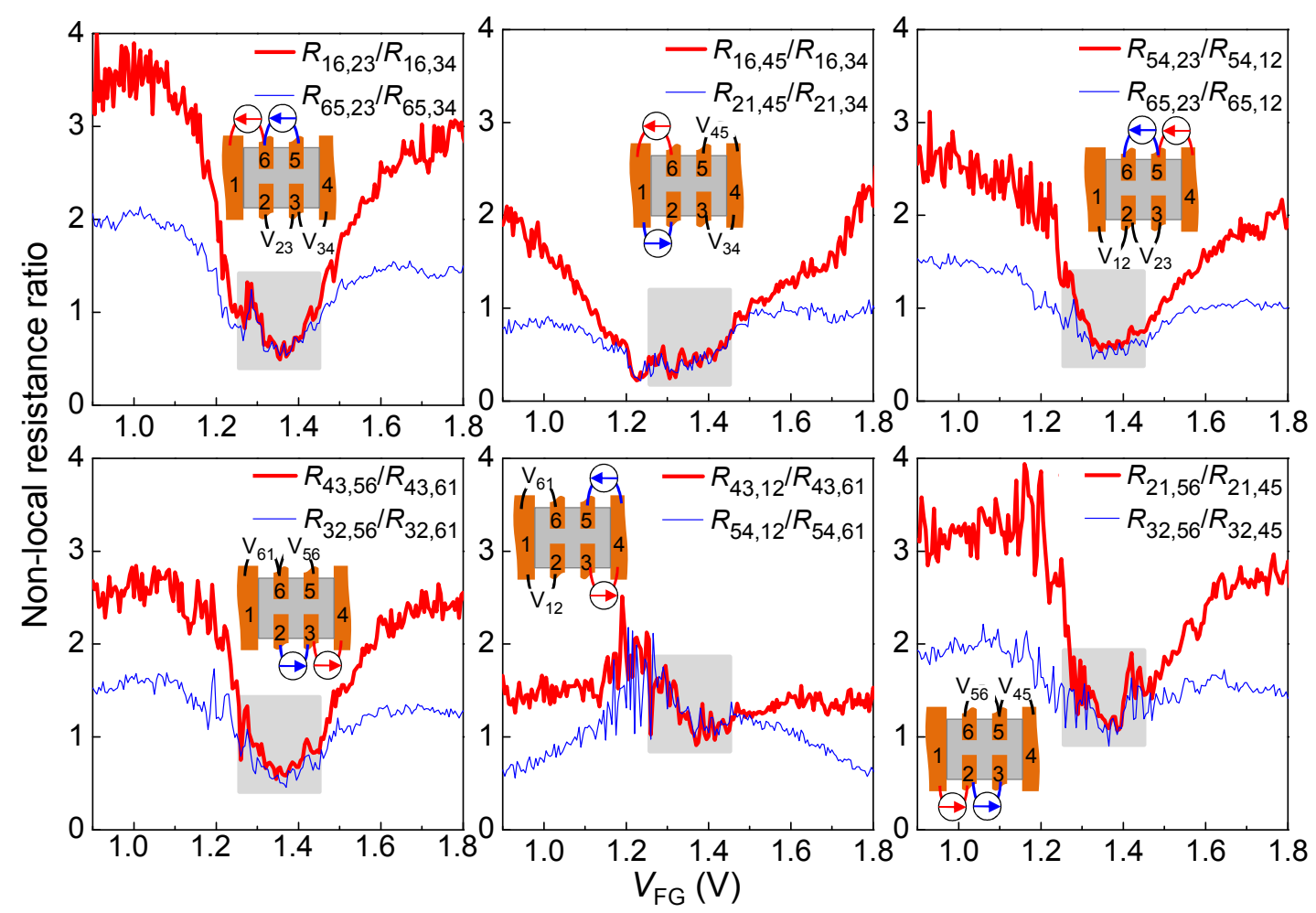

FIG. 4: (Color online) Results of non-local measurements on the $w=12 \mathrm{~nm}$ sample for various current/voltage contact configurations at $0.25 \mathrm{~K}$. Each panel corresponds to a given set of adjacent voltage-probe pairs shown in the inset. Ratio between non-local resistances measured with these voltage-probe pairs are plotted as a function of $V_{\mathrm{FG}}$. The two traces in each panel represent measurements for the two different current injection/ejection paths shown in the inset. Note that each measurement uses only one of the two current sources shown in the inset. The gray background represents the region where the Fermi level is expected to be in the gap.

ingly, the non-local resistances exhibit sharp fluctuations as a function of $V_{\mathrm{FG}}$. These resistance fluctuations are reproducible with amplitudes larger than the background noise level (see Appendix).

As we show below, focusing on the ratio between nonlocal resistances measured for adjacent voltage-probe pairs reveals that transport is dominated by edge channels despite the significant fluctuations and deviation from the quantized value. In Fig. 3(d), we plot resistance ratios $R_{16,23} / R_{16,34}$ and $R_{65,23} / R_{65,34}$ calculated from the data in Figs. 3(b) and 3(c) as a function of $V_{\mathrm{FG}}$. Physically, these ratios represent the voltage ratio $V_{23} / V_{34}$ that appears for a given current injection/ejection path, 1-6 or 6-5. If bulk transport is dominant, the voltage ratio $V_{23} / V_{34}$ would simply reflect the current distribution inside the bulk, which is determined solely by the current injection/ejection path and does not depend on sample conductivity. Therefore, the ratios should take different values for different current paths. This is actually seen in the $V_{\mathrm{FG}}$ ranges where the Fermi level is outside the gap; at $V_{\mathrm{FG}}<1.25 \mathrm{~V}$ ( $p$-type region) and $V_{\mathrm{FG}}>1.45 \mathrm{~V}$ ( $n$-type region), $R_{16,23} / R_{16,34}$ and $R_{65,23} / R_{65,34}$ do not coincide with each other.

In contrast, if edge transport is dominant as in Fig. 3(a), the voltage ratio $V_{23} / V_{34}$ should directly re- flect the relative magnitude of the resistances $r_{23}$ and $r_{34}$ of the relevant edge channels, since the same current $\left(i_{1}\right.$ or $\left.i_{1}^{\prime}\right)$ flows through them for a given current injection/ejection path [Fig. 3(a)]. An important corollary of the edge transport is that the ratio $V_{23} / V_{34}=r_{23} / r_{34}$ is an intrinsic property of the sample and therefore does not depend on the current injection/ejection path. Note that this holds for arbitrary values of $r_{j, j+1}$, that is, even if they deviate from the quantized value and consequently the current partition ratio deviates from 1:5, provided that the bulk transport is negligible. Indeed, we observe that in the gap region $\left(1.25<V_{\mathrm{FG}}<1.45 \mathrm{~V}\right)$ the resistance ratios for the two current paths coincide with each other [Fig. 3(d)], including the fine details of the fluctuations [Fig. 3(e)].

Figure 4 compiles the results of similar non-local measurements on the same sample for all possible contact configurations. In each panel, the ratio between the nonlocal resistances measured with a given set of adjacent voltage-probe pairs is plotted as a function of $V_{\mathrm{FG}}$. The two traces in each panel represent the results obtained for the two different current injection/ejection paths shown in the inset. The data demonstrate that, for all configurations, the resistance ratios become independent of the current path in the gap region. These results constitute 
compelling evidence that the transport in the gap region is dominated by edge channels.

Figure 3(f) shows the resistance ratios measured at 4.3 $\mathrm{K}$ for the same contact geometries as in Fig. 3(d) at 0.25 $\mathrm{K}$. The behavior outside the gap region $\left(V_{\mathrm{FG}}<1.25 \mathrm{~V}\right.$ and $V_{\mathrm{FG}}>1.45 \mathrm{~V}$ ) is similar to that at $0.25 \mathrm{~K}$, as expected for the bulk conduction. On the other hand, in the gap region, the resistance ratios for the two current paths no longer coincide with each other. This indicates that at high temperatures there is a finite current that flows directly between non-adjacent contacts through the bulk region. This is reasonable because at $4.3 \mathrm{~K}$ thermally activated carriers contribute to the bulk conduction; it is also consistent with the temperature dependence of $R_{x x}$ [Fig. 2(b)].

By comparing a series of InAs/GaSb devices with different lengths and widths, Knez and co-workers were able to separate out the contributions of bulk and edge transport; they found that there was a significant contribution from the bulk conductivity $g_{\text {bulk }} \approx 5 e^{2} / h$ at $0.3 \mathrm{~K}$ in their samples $\frac{19}{\underline{19}}$ Assuming the same bulk conductivity for our sample geometry would yield $R_{14,23}=1.5 \mathrm{k} \Omega$, which is much smaller than the measured value for the $w=12 \mathrm{~nm}$ sample. This clearly demonstrates that, by appropriately designing the layer structure, the InAs/GaSb system can be tailored to have conducting edge channels while keeping a gap in the bulk region, as predicted in Ref. 15 .

Owing to the fact that bulk conduction in our sample is negligible at low temperatures, we are able to determine the resistance ratios for all the adjacent contact pairs by performing non-local measurements while sequentially changing the current/voltage contact configurations. This allows us to extract the individual resistances of all the edge channels as a function of $V_{\mathrm{FG}}$ [Fig. 5(a)]. The channel resistances deduced in this way range from 13 to $70 \mathrm{k} \Omega$, which deviate greatly from the expected quantized value, $h / e^{2} \approx 25.8 \mathrm{k} \Omega$. To verify the consistency of measurements, the longitudinal resistance $R_{14,65}$ is reconstructed from these channel resistances using the circuit model in Fig. 3(a). Despite the large scattering of the individual channel resistances and experimental errors involved, the reconstructed $R_{14,65}$ agrees well with measured value [Fig. 5(b)].

As the data in Fig. 5(a) demonstrate, non-local measurements allow us to examine the scattering processes occurring in a single edge channel. The resistances exhibit fluctuations indicative of energy-dependent inelastic scattering. The amplitude of the fluctuations ranges up to about $h / e^{2}$. Inelastic scattering can occur when there are electron or hole puddles arising from spatial potential fluctuations near the helical edge channels that serve as reservoirs in the same way as ohmic contacts do. Hence these puddles increase the resistance in the same way as additional ohmic contacts embedded along the edge channels ${ }^{12,13}$ It is possible that the observed $V_{\mathrm{FG}^{-}}$ dependent fluctuations represent the charging effects of localized puddles that act as scatterers by exchanging electrons with the edge channels. We should note that
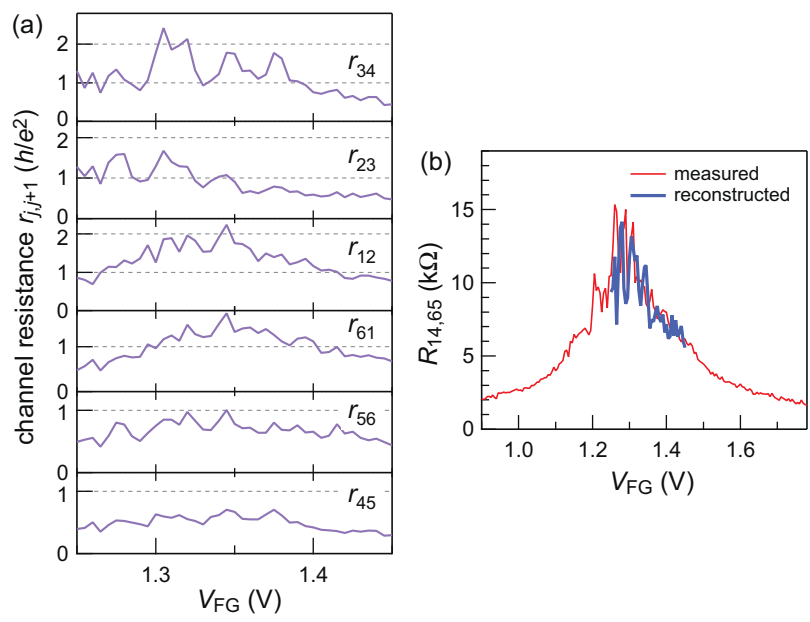

FIG. 5: (Color online) (a) Resistances of individual edge channels deduced from non-local resistance measurements at 0.25 K. (b) Comparison of longitudinal resistance $R_{14,65}$ reconstructed from the individual channel resistances shown in (a) with that measured directly.

these scatterers always act to increase the channel resistance. On the other hand, if there is a large puddle that is capable of exchanging carriers with both of two adjacent contacts, it will act to decrease the channel resistance; this might explain the result where $r_{45}$ and $r_{56}$ are smaller than $h / e^{2}$. Figure $5\left(\right.$ a) also shows that the $V_{\mathrm{FG}}$ range that satisfies $r_{j, j+1} \geq h / e^{2}$ varies slightly with the position of the edge, suggesting that long-range sample inhomogeneity makes it difficult to tune the Fermi level in the gap region over the entire sample area. Further investigations are necessary to clarify the exact scattering mechanism.

\section{SUMMARY}

In summary, we have investigated transport in $\mathrm{InAs} / \mathrm{GaSb}$ heterostructures with different InAs layer thicknesses. From systematic non-local resistance measurements, we have shown that a sample with 12-nmthick InAs layer has a bulk gap and edge channel transport. Our method offers a way of elucidating the properties of $2 \mathrm{D}$ TIs even when the inelastic mean free path is shorter than the edge channel length and quantized transport is absent. 


\section{Acknowledgments}

We thank Yoshito Ishikawa, Satoshi Sasaki, and Mineo Ueki for their help with the experiments.

\section{Appendix}

The resistance fluctuation observed for the $w=12 \mathrm{~nm}$ sample reflects the change in gate voltage. This can be verified by comparing it with the time-dependent fluctuation of the signal. Figure 6(a) shows the non-local resistance $R_{32,45}$ as a function of $V_{\mathrm{FG}}$, where the resistance was monitored at each $V_{\mathrm{FG}}$ for $60 \mathrm{sec}$ with a 1sec interval. The trace represents the average taken at each $V_{\mathrm{FG}}$ and the vertical bars indicate the amplitude of the time-dependent fluctuation at each $V_{\mathrm{FG}}$. The dashed rectangular region in Fig. 6(a) is enlarged in Fig. 6(b). It can be seen that the amplitude of the time-dependent fluctuation including background noise is much smaller than that of the $V_{\mathrm{FG}}$-dependent resistance fluctuations.

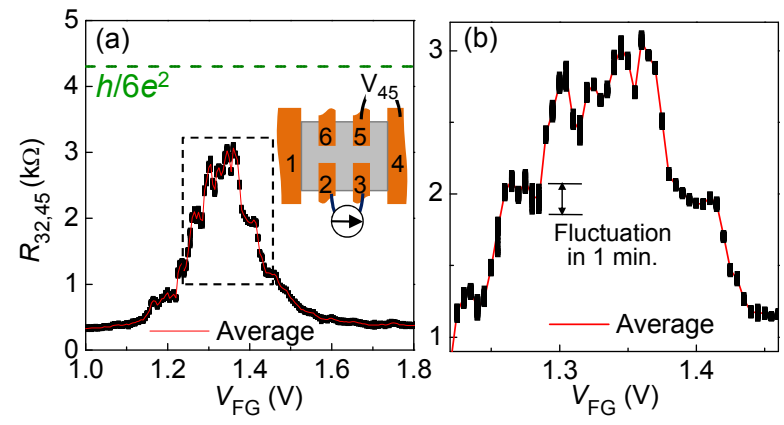

FIG. 6: (Color online) (a) Non-local resistance $R_{32,45}$ as a function of $V_{\mathrm{FG}}$ for the $w=12 \mathrm{~nm}$ sample $(T=0.25 \mathrm{~K})$. The trace represents the average taken over $1 \mathrm{~min}$. at each $V_{\mathrm{FG}}$ and the vertical bars indicate the amplitude of the timedependent fluctuation at each $V_{\mathrm{FG}}$. Inset shows the measurement configuration. (b) Magnified view of the data shown in the dashed rectangle in (a).
* Electronic address: suzuki.kyoichi@lab.ntt.co.jp

1 C. L. Kane and E. J. Mele, Phys. Rev. Lett. 95,146802 (2005).

2 C. L. Kane and E. J. Mele, Phys. Rev. Lett. 95,226801 (2005).

3 B. A. Bernevig and S.-C. Zhang, Phys. Rev. Lett. 96, 106802 (2006).

4 M. Z. Hasan and C. L. Kane, Rev. Mod. Phys.82, 3045 (2010) and references therein.

5 X.-L. Qi and S.-C. Zhang, Rev. Mod. Phys. 83, 1057 (2011) and references therein.

6 T. Sato, K. Segawa, H. Guo, K. Sugawara, S. Souma, T. Takahashi, and Y. Ando, Phys. Rev. Lett., 105, 136802 (2010);

7 K. Kuroda, M. Ye, A. Kimura, S. V. Eremeev, E. E. Krasovskii, E. V. Chulkov, Y. Ueda, K. Miyamoto, T. Okuda, K. Shimada, H. Namatame, and M. Taniguchi, Phys. Rev. Lett. 105, 146801 (2010).

8 B. A. Bernevig, T. L. Hughes, and S-C.Zhang, Science 314, 1757 (2006).

9 M. König, S. Wiedmann, C. Brüne, A. Roth, H. Buhmann, L. W. Molenkamp, X.-L. Qi, S.-C. Zhang, Science 318, 766 (2007)

10 M. König, H. Buhmann, L. W. Molenkamp, T. Hughes, C.-X. Liu, X.-L. Qi, and S.-C. Zhang, J. Phys. Soc. Jpn. 77, 031007 (2008).

11 A. Roth, C. Brüne, H. Buhmann, L. W. Molenkamp, J. Maciejko, X.-L. Qi, S.-C. Zhang, Science 325, 294 (2009).

12 Supporting online material of Ref. [11].
13 J. I. Väyrynen, M. Goldstein, and L. I. Glazman, Phys. Rev. Lett. 110, 216402 (2013).

14 S. Murakami, New J. Phys. 13, 105007 (2011).

15 C. Liu, T. L. Hughes, X.-L. Qi, K.Wang, and S.-C. Zhang, Phys. Rev. Lett. 100, 236601 (2008).

16 M. Altarelli, Phys. Rev. B 28, 842 (1983).

17 K. Suzuki, K. Takashina, S. Miyashita, and Y. Hirayama, Phys. Rev. Lett. 93, 016803 (2004).

18 J. Li, W. Yang, and K. Chang, Phys. Rev. B 80, 035303 (2009).

19 I. Knez, R. R. Du, and G. Sullivan, Phys. Rev. Lett. 107, 136603 (2011).

20 C. Xu and J. E. Moore Phys. Rev. B 73, 045322 (2006).

21 C. Wu, B. A. Bernevig, and S.-C. Zhang, Phys. Rev. Lett. 96, 106401, (2006).

22 J. Maciejko, C.-X Liu, Y. Oreg, X.-L. Qi, C. Wu, and S.-C. Zhang, Phys. Rev. Lett. 102, 256803 (2009).

23 J. C. Budich, F. Dolcini, P. Recher, and B. Trauzettel, Phys. Rev. Lett. 108, 086602 (2012).

24 T. L. Schmidt, S. Rachel, F. von Oppen, and L. I. Glazman Phys. Rev. Lett. 108, 156402 (2012).

25 C. Kadow, H.-K. Lin, M. Dahlstroem, M. Rodwell, A. C. Gossard, B. Brar, and G. Sullivan, J. Cryst. Growth 251, 543 (2003).

26 K. Suzuki, Y. Harada, F. Maeda, K. Onomitsu, T. Yamaguchi, and K. Muraki, Appl. Phys. Express 4, 125702 (2011). 